\title{
Translation surfaces in affine 3-space
}

\author{
Mohamd Saleem Lone*1 (D), Murat Kemal Karacan² (D), Yilmaz Tuncer² (D), \\ Hasan $\mathrm{Es}^{3}$ (iD \\ ${ }^{1}$ International Centre for Theoretical Sciences, Tata Institute of Fundamental Research, 560089, \\ Bengaluru, India \\ ${ }^{2}$ Usak University, Faculty of Sciences and Arts, Department of Mathematics, 1 Eylul Campus, \\ 64200, Usak, Turkey \\ ${ }^{3}$ Gazi University, Gazi Educational Faculty, Department of Mathematical Education, 06500 Teknikokullar \\ Ankara, Turkey
}

\begin{abstract}
In this paper, we study translation surfaces in three dimensional affine space. We characterize the finite type non-degenerate translation surfaces with respect to the first affine and the second affine fundamental forms.
\end{abstract}

Mathematics Subject Classification (2010). 53A35, 53B30, 53A40

Keywords. affine space, Laplace operator, translation surfaces

\section{Introduction}

A surface obtained by translating a curve $\alpha(u)$ over another curve $\beta(v)$ is called a translation surface, i.e., $z(u, v)=\varphi(u)+\phi(v)$. Translation surfaces are used by the architects in the designing and construction of glass roof structures $([3,10])$.

H. F. Scherk in 1835 proved that, in addition to the planes, the only minimal translation surfaces are given by

$$
z(u, v)=\frac{1}{c} \log |\cos (c u)|-\frac{1}{c} \log |\cos (c v)|,
$$

where $c$ is a non-zero constant ([21]). The study of translation surfaces in affine spaces was initially started bt Manhart ([16]), Magid and Vrancken ([15]). Manhart gave a complete classification of non-degenerate minimal translation surfaces in affine space, while as Magid and Vrancken classified the flat translation surfaces. For more study of surfaces (translation) in affine spaces, we refer the reader to see $([1,2,11,12,18-20])$.

Now, we give a brief description of another direction of geometry known as finite type of surfaces (submanifolds). A surface or a submanifold is said to be of finite type or finite Chen type if its coordinate functions are a finite sum of eigenfunctions of its Laplacian. Let $x$ and $H$ be the position vector and the mean curvature of a $n$-dimensional submanifold $\left(M^{n}\right)$ of $\mathbb{E}^{m}$, we know from a well known formula ([7]) $\Delta x=-n H$. This implies that $M^{n}$ is minimal if and only if the coordinate functions are harmonic or they

\footnotetext{
* Corresponding Author.

Email addresses: saleemraja2008@gmail.com (M.S. Lone), murat.karacan@usak.edu.tr (M.K. Karacan), yilmaz.tuncer@usak.edu.tr (Y. Tuncer), hasanes@gazi.edu.tr (H. Es)

Received: 14.05.2019; Accepted: 27.02.2020
} 
are the eigenfunctions of $\Delta$ with eigenvalue being zero. It was Takahashi who first proved that the submanifolds satisfying $\Delta x=-\lambda x$ with identical $\lambda \in \mathbb{R}$ are either minimal submanifolds of $\mathbb{E}^{m}$ or minimal submanifolds of hyperspheres $S^{m-1}$ of $\mathbb{E}^{m}$. For more study of finite type of surfaces (submanifolds), we refer $([4,8,9,13,21])$.

As of now, there is no study available where the authors may have investigated finite type of surfaces in affine spaces. So the aim of this paper is to connect finite type of study with the affine geometry. Apart from revisiting the minimal and flat translation surfaces, the main objective of this paper is to classify the finite type of translation surfaces in affine space with respect to first and second fundamental forms.

\section{Preliminaries}

In this section, we will introduce some basic notions of affine structures: the co-normal and normal vectors, Gaussian and the mean curvatures. In case of affine transformations, the Berwald-Blaschke metric is an invariant quadratic form and independent of coordinate systems. It is not necessary that this quadratic form is positive definite. Let $\Phi: \Omega \rightarrow \mathbb{R}^{3}$ be a regular surface, the Berwald-Blaschke metric is given by

$$
\begin{aligned}
h & =\frac{L}{\left|L N-M^{2}\right|^{\frac{1}{4}}} d u^{2}+\frac{2 M}{\left|L N-M^{2}\right|^{\frac{1}{4}}} d u d v+\frac{N}{\left|L N-M^{2}\right|^{\frac{1}{4}}} d v^{2} \\
& =E d u^{2}+2 F d u d v+G d v^{2},
\end{aligned}
$$

where

$$
L=\left[\Phi_{u}, \Phi_{v}, \Phi_{u u}\right], \quad N=\left[\Phi_{u}, \Phi_{v}, \Phi_{v v}\right], \quad M=\left[\Phi_{u}, \Phi_{v}, \Phi_{u v}\right] .
$$

From now onwards, we suppose, $L N-M^{2} \neq 0$, i.e., $\Phi$ is non-degenerate. The points where $L N-M^{2}$ is positive, negative or zero are called elliptical, hyperbolic or parabolic, respectively $([1,2,5,11])$. In this paper, we are studying only in convex case (elliptical points).

Definition 2.1. Let $\mathbf{X}$ be a regular surface given by the parametrization $\Phi: \Omega \subset \mathbb{R}^{2} \rightarrow$ $\mathbf{X} \subset \mathbb{R}^{3}$, the affine conormal field is given by

$$
\eta=\frac{\Phi_{u} \wedge \Phi_{v}}{\left|L N-M^{2}\right|^{\frac{1}{4}}}=\frac{\Phi_{u} \wedge \Phi_{v}}{\left[\eta, \eta_{u}, \eta_{v}\right]},
$$

where $L, N$ and $M$ are the coefficients of the first affine fundamental form.

By definition, it can be seen that $\eta \cdot d \Phi=0$. Let $\sigma= \pm\left[\eta, \eta_{u}, \eta_{v}\right]= \pm\left(L N-M^{2}\right)^{\frac{1}{4}}$, where the sign \pm depends on whether the points are elliptical or hyperbolic. Thus, we can write

$$
\eta=\frac{\Phi_{u} \wedge \Phi_{v}}{\sigma}
$$

Definition 2.2. Let $\mathbf{X}$ be a regular surface given by the parametrization $\Phi: \Omega \subset \mathbb{R}^{2} \rightarrow$ $\mathbf{X} \subset \mathbb{R}^{3}$, the affine normal vector field is given by

or

$$
\zeta=\frac{\eta_{u} \wedge \eta_{v}}{\left[\eta, \eta_{u}, \eta_{v}\right]}=\frac{\eta_{u} \wedge \eta_{v}}{\sigma}
$$

Or

$$
\zeta=\frac{1}{2} \frac{\left|L N-M^{2}\right|^{\frac{1}{4}}}{\sqrt{L N-M^{2}}}\left[\frac{\partial}{\partial u}\left(\frac{N \Phi_{u}-M \Phi_{v}}{\sqrt{L N-M^{2}}}\right)+\frac{\partial}{\partial v}\left(\frac{L \Phi_{v}-M \Phi_{u}}{\sqrt{L N-M^{2}}}\right)\right]
$$

$$
\zeta=\frac{1}{2} \frac{1}{\sqrt{E G-F^{2}}}\left[\frac{\partial}{\partial u}\left(\frac{G \Phi_{u}-F \Phi_{v}}{\sqrt{E G-F^{2}}}\right)+\frac{\partial}{\partial v}\left(\frac{E \Phi_{v}-F \Phi_{u}}{\sqrt{E G-F^{2}}}\right)\right],
$$


where

$$
\eta \cdot \zeta=1, \quad \eta_{u} \cdot \zeta=0, \quad \eta_{v} . \zeta=0 .
$$

We see that the affine normal vector field does not belong to the tangent plane of $\mathbf{X}$.

The affine curvature is given by the variation of the normal vector field. Since $\eta \cdot \zeta_{u}=$ $0, \quad \eta$. $\zeta_{v}=0$, i.e., $\zeta_{u}$ and $\zeta_{v}$ are orthogonal to $\eta$. In particular $\zeta_{u}, \zeta_{v} \in T_{p} \mathbf{X}$ (tangent plane of $\mathbf{X}$ at $p$.) Therefore, the shape operator $\left(S: T_{p} \mathbf{X} \rightarrow T_{p} \mathbf{X}\right)$ at a point $p$ is given by

$$
S_{p}(v)=-D_{v} \zeta \text {. }
$$

Since $\zeta_{u}, \zeta_{v} \in T_{p}(\mathbf{X})$, we have

$$
b_{i j}: \Omega \rightarrow \mathbb{R}, i, j=1,2,
$$

such that

$$
\left\{\begin{array}{l}
\zeta_{u}=b_{11} \Phi_{u}+b_{12} \Phi_{v} \\
\zeta_{v}=b_{21} \Phi_{u}+b_{22} \Phi_{v}
\end{array}\right.
$$

where

$$
\begin{cases}b_{11}=\frac{\left[\zeta_{u}, \Phi_{v}, \zeta\right]}{\sigma}, & b_{12}=\frac{\left[\zeta_{v}, \Phi_{v}, \zeta\right]}{\sigma}, \\ b_{21}=\frac{\left[\Phi_{u}, \zeta_{u}, \zeta\right]}{\sigma}, & b_{22}=\frac{\left[\Phi_{u}, \zeta_{v}, \zeta\right]}{\sigma} .\end{cases}
$$

Notice that, in case of affine geometry, the corresponding matrix $B=\left(b_{i j}\right),\{i, j=1,2\}$ of the shape operator is not necessarily symmetric $([1,2,5,11])$.

The affine Gaussian curvature and the affine mean curvature are given by

$$
\mathbf{K}=\operatorname{det} B=b_{11} b_{22}-b_{12} b_{21}, \quad \mathbf{H}=\frac{1}{2} \operatorname{tr} B=\frac{b_{11}+b_{22}}{2},
$$

respectively.

The Laplacian operators $\Delta^{I}$ and $\Delta^{h}$ of the first affine and the second affine fundamental forms on $\mathbf{X}$ with respect to local coordinates $\{u, v\}$ are defined by $([6,20])$ :

$$
\Delta^{I} \Phi=-\frac{1}{\sqrt{L N-M^{2}}}\left[\frac{\partial}{\partial u}\left(\frac{N \Phi_{u}-M \Phi_{v}}{\sqrt{L N-M^{2}}}\right)-\frac{\partial}{\partial v}\left(\frac{M \Phi_{u}-L \Phi_{v}}{\sqrt{L N-M^{2}}}\right)\right]
$$

and

$$
\Delta^{h} \Phi=-\frac{1}{\sqrt{E G-F^{2}}}\left[\frac{\partial}{\partial u}\left(\frac{G \Phi_{u}-F \Phi_{v}}{\sqrt{E G-F^{2}}}\right)-\frac{\partial}{\partial v}\left(\frac{F \Phi_{u}-E \Phi_{v}}{\sqrt{E G-F^{2}}}\right)\right],
$$

respectively. For more study of affine spaces, we refer $[14,17]$.

\section{Translation surfaces in affine 3 -space}

A surface $\mathbf{X}$ obtained as the sum of two affine curves $\alpha(u)=(u, 0, \varphi(u))$ and $\beta(v)=$ $(0, v, \phi(v))$ is called a translation surface in affine 3 -space. Thus, a translation surface is defined as a graph

$$
\mathbf{X}: \Phi(u, v)=(u, v, \varphi(u)+\phi(v)) .
$$

A basis for the tangent vectors is given by

$$
\Phi_{u}=\left(1,0, \varphi^{\prime}(u)\right), \quad \Phi_{v}=\left(0,1, \phi^{\prime}(v)\right) .
$$

The second order partial derivatives of $\Phi(u, v)$ are given by

$$
\Phi_{u u}=\left(0,0, \varphi^{\prime \prime}(u)\right), \quad \Phi_{u v}=(0,0,0), \quad \Phi_{v v}=\left(0,0, \phi^{\prime \prime}(v)\right) .
$$

The coefficients of the first affine fundamental form are given by

$$
L=\varphi^{\prime \prime}(u), N=\phi^{\prime \prime}(v), M=0, \quad \sigma=\left(L N-M^{2}\right)^{\frac{1}{4}}=\left(\varphi^{\prime \prime} \phi^{\prime \prime}\right)^{\frac{1}{4}} .
$$


Hence the coefficients of the Berwald-Blaschke metric of the translation surface or the coefficients of the second affine fundamental form are given by

$$
E=\frac{\varphi^{\prime \prime}}{\left(\varphi^{\prime \prime} \phi^{\prime \prime}\right)^{\frac{1}{4}}}, G=\frac{\phi^{\prime \prime}}{\left(\varphi^{\prime \prime} \phi^{\prime \prime}\right)^{\frac{1}{4}}}, F=0 .
$$

Since we are supposing that the Berwald-Blaschke metric is non-degenerate: $\sigma \neq 0$. Geometrically $\sigma>0$ means that the Euclidean Gaussian curvature does not vanish, i.e., the translation surface is strongly convex. The affine co-normal field of the translation surface is given by

$$
\eta=\left(-\frac{\varphi^{\prime}}{\left(\varphi^{\prime \prime} \phi^{\prime \prime}\right)^{\frac{1}{4}}},-\frac{\phi^{\prime}}{\left(\varphi^{\prime \prime} \phi^{\prime \prime}\right)^{\frac{1}{4}}}, \frac{1}{\left(\varphi^{\prime \prime} \phi^{\prime \prime}\right)^{\frac{1}{4}}}\right) .
$$

Thus, the affine normal vector field is given by

$$
\zeta=\left(-\frac{\varphi^{\prime \prime \prime}\left(\varphi^{\prime \prime} \phi^{\prime \prime}\right)^{\frac{1}{4}}}{4 \varphi^{\prime \prime 2}}, \quad-\frac{\phi^{\prime \prime \prime}\left(\varphi^{\prime \prime} \phi^{\prime \prime}\right)^{\frac{1}{4}}}{4 \phi^{\prime \prime^{2}}}, \quad \frac{\left(\varphi^{\prime \prime} \phi^{\prime \prime}\right)^{\frac{1}{4}}\left(-\varphi^{\prime} \phi^{\prime \prime^{2}} \varphi^{\prime \prime \prime}+\varphi^{\prime \prime^{2}}\left(4 \phi^{\prime{ }^{2}}-\phi^{\prime} \phi^{\prime \prime \prime}\right)\right)}{4 \varphi^{\prime \prime 2} \phi^{\prime \prime 2}}\right) .
$$

Consequently, the coefficients of the matrix $B=\left(b_{i j}\right)$ are given by

$$
\begin{aligned}
& b_{11}=\frac{\left(\varphi^{\prime \prime} \phi^{\prime \prime}\right)^{\frac{1}{4}}\left(7 \varphi^{\prime \prime \prime}-4 \varphi^{\prime \prime} \varphi^{(4)}\right)}{16 \varphi^{\prime \prime^{3}}}, \quad b_{12}=-\frac{\phi^{\prime \prime} \varphi^{\prime \prime \prime} \phi^{\prime \prime \prime}}{16\left(\varphi^{\prime \prime} \phi^{\prime \prime}\right)^{\frac{7}{4}}}, \\
& b_{21}=-\frac{\varphi^{\prime \prime} \varphi^{\prime \prime \prime} \phi^{\prime \prime \prime}}{16\left(\varphi^{\prime \prime} \phi^{\prime \prime}\right)^{\frac{7}{4}}}, \quad b_{22}=\frac{\left(\varphi^{\prime \prime} \phi^{\prime \prime}\right)^{\frac{1}{4}}\left(7 \phi^{\prime \prime \prime}{ }^{2}-4 \phi^{\prime \prime} \phi^{(4)}\right)}{16 \phi^{\prime \prime^{3}}} .
\end{aligned}
$$

Therefore from (2.9), we can easily find the affine Gaussian [12] and the affine mean curvatures [18] of $\mathbf{X}$ are given by

$$
\left\{\begin{array}{l}
\mathbf{K}=\frac{\varphi^{\prime \prime \prime}\left(12 \phi^{\prime \prime \prime}{ }^{2}-7 \phi^{\prime \prime} \phi^{(4)}\right)+\varphi^{\prime \prime} \varphi^{(4)}\left(-7{\phi^{\prime \prime \prime}}^{2}+4 \phi^{\prime \prime} \phi^{(4)}\right)}{64\left(\varphi^{\prime \prime} \phi^{\prime \prime}\right)^{\frac{5}{2}}}, \\
\mathbf{H}=\left(\frac{\left(\varphi^{\prime \prime} \phi^{\prime \prime}\right)^{\frac{1}{4}}\left(7 \varphi^{\prime \prime \prime}{ }^{2}-4 \varphi^{\prime \prime} \varphi^{(4)}\right)}{32{\varphi^{\prime \prime}}^{3}}+\frac{\left(\varphi^{\prime \prime} \phi^{\prime \prime}\right)^{\frac{1}{4}}\left(7{\phi^{\prime \prime \prime}}^{2}-4 \phi^{\prime \prime} \phi^{(4)}\right)}{32 \phi^{\prime \prime}}\right),
\end{array}\right.
$$

respectively.

Suppose that the translation surface given by (3.1) has zero affine Gaussian curvature, we have

$$
\varphi^{\prime \prime \prime \prime^{2}}\left(12 \phi^{\prime \prime \prime}{ }^{2}-7 \phi^{\prime \prime} \phi^{(4)}\right)+\varphi^{\prime \prime} \varphi^{(4)}\left(-7 \phi^{\prime \prime \prime}{ }^{2}+4 \phi^{\prime \prime} \phi^{(4)}\right)=0 .
$$

Here $u$ and $v$ are two independent variables, so each side of (3.11) is equal to a constant $p \in \mathbb{R}$. Hence, the equation (3.11) is reduced to

$$
\frac{\varphi^{\prime \prime \prime}}{\varphi^{\prime \prime} \varphi^{(4)}}=p=\frac{7 \phi^{\prime \prime \prime}{ }^{2}-4 \phi^{\prime \prime} \phi^{(4)}}{12 \phi^{\prime \prime \prime}-7 \phi^{\prime \prime} \phi^{(4)}} \text {. }
$$

By solving (3.12), we get

$$
\varphi(u)=c_{1}+c_{2} u-\frac{c_{3}\left(u-p u+c_{4} p\right)^{\frac{3 p-2}{p-1}}}{6 p^{2}-7 p+2}, \quad \phi(v)=c_{5}+c_{6} v-\frac{c_{7}\left((5 p-3) v+c_{8}(7 p-4)\right)^{\frac{2-3 p}{3-5 p}}}{6 p^{2}-7 p+2}
$$


for some constants $c_{i} \in \mathbb{R}$ and $p \neq 1, \frac{3}{5}$. Thus $\mathbf{X}$ is parametrized by

$\Phi(u, v)=\left(u, v,\left(c_{1}+c_{2} u-\frac{c_{3}\left(u-p u+c_{4} p\right)^{\frac{3 p-2}{p-1}}}{6 p^{2}-7 p+2}\right)+\left(c_{5}+c_{6} u-\frac{c_{7}\left((5 p-3) v+c_{8}(7 p-4)\right)^{\frac{2-3 p}{3-5 p}}}{6 p^{2}-7 p+2}\right)\right)$.

In particular, if $p=0$, we have

$$
\varphi(u)=c_{1} u^{2}+c_{2} u+c_{3}, \quad \phi(v)=c_{4}+c_{5} v-\frac{c_{6}\left(3 v+4 c_{7}\right)^{\frac{2}{3}}}{2},
$$

where $c_{i} \in \mathbb{R}$. In this case, $\mathbf{X}$ is parametrized by

$$
\Phi(u, v)=\left(u, v,\left(c_{1} u^{2}+c_{2} u+c_{3}\right)+\left(c_{4}+c_{5} v-\frac{c_{6}\left(3 v+4 c_{7}\right)^{\frac{2}{3}}}{2}\right)\right) .
$$

Theorem 3.1. Let $\boldsymbol{X}$ be a translation surface in affine 3-space. If $\boldsymbol{X}$ has zero Gaussian curvature or affine flat, then $\boldsymbol{X}$ is parameterized by (3.14) or (3.16).

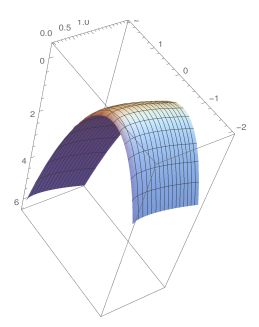

Figure 1. 3D plot of (3.16) for particular values of $c_{i} \in \mathbb{R}$.

Remark 3.2. For $p=1$, the equations in (3.12) reduces to

$$
\varphi^{\prime \prime \prime 2}-\varphi^{\prime \prime} \varphi^{(4)}=0, \quad 5 \phi^{\prime \prime \prime 2}-3 \phi^{\prime \prime} \phi^{(4)}=0 .
$$

Solving (3.17), we get

$$
\varphi(u)=c_{1} \frac{e^{u c_{2}}}{c_{2}^{2}}+c_{3} u+c_{4}, \quad \phi(v)= \pm c_{5}\left(2 v+3 c_{6}\right)^{\frac{1}{2}}+c_{7} v+c_{8},
$$

where $c_{i} \in \mathbb{R}$. Set $c_{1}=c_{2}=1, c_{5}=\frac{1}{\sqrt{2}}, c_{3}=c_{4}=c_{6}=c_{7}=c_{8}=0$ in (3.18), we get $\varphi(u)=e^{u}$ and $\phi(v)= \pm v^{\frac{1}{2}}$. Hence the surface parameterization is

$$
\Phi(u, v)=\left(u, v, e^{u} \pm v^{\frac{1}{2}}\right),
$$

which is (1.2) of theorem 1.1 in [12]. Similarly, fixing properly the constants in theorem (3.1), we can easily find the other results of theorem 1.1 in [12].

Now assume that $\mathbf{X}$ is affine minimal. Hence, the mean curvature is zero if and only if

$$
\frac{\left(\varphi^{\prime \prime} \phi^{\prime \prime}\right)^{\frac{1}{4}}\left(7 \varphi^{\prime \prime \prime} \prime^{2}-4 \varphi^{\prime \prime} \varphi^{(4)}\right)}{32 \varphi^{\prime \prime \prime}}+\frac{\left(\varphi^{\prime \prime} \phi^{\prime \prime}\right)^{\frac{1}{4}}\left(7 \phi^{\prime \prime \prime^{2}}-4 \phi^{\prime \prime} \phi^{(4)}\right)}{32{\phi^{\prime \prime}}^{3}}=0 .
$$

The minimality condition (3.20) can be separated for the variables as:

$$
\frac{\left(7 \varphi^{\prime \prime \prime^{2}}-4 \varphi^{\prime \prime} \varphi^{(4)}\right)}{32 \varphi^{\prime \prime^{3}}}=p=-\frac{\left(7{\phi^{\prime \prime \prime}}^{2}-4 \phi^{\prime \prime} \phi^{(4)}\right)}{32{\phi^{\prime \prime}}^{3}} .
$$

In this case, the nonlinear differential equation (3.21) has not any real analytic solution for $p \in \mathbb{R} \backslash\{0\}$. For $p=0$, we have

$$
\varphi(u)=c_{1}+c_{2} u-\frac{c_{3}\left(3 u+4 c_{4}\right)^{\frac{2}{3}}}{2}, \quad \phi(v)=c_{5}+c_{6} v-\frac{c_{7}\left(3 v+4 c_{8}\right)^{\frac{2}{3}}}{2},
$$


for constants $c_{i} \in \mathbb{R}$. In this case, $\mathbf{X}$ is parametrized by

$$
\Phi(u, v)=\left(u, v,\left(c_{1}+c_{2} u-\frac{c_{3}\left(3 u+4 c_{4}\right)^{\frac{2}{3}}}{2}\right)+\left(c_{5}+c_{6} v-\frac{c_{7}\left(3 v+4 c_{8}\right)^{\frac{2}{3}}}{2}\right)\right) .
$$

Theorem 3.3. A translation surface $\boldsymbol{X}$ is affine minimal in affine 3-space if and only if it is a part of the surface (3.23).

Remark 3.4. Choose $c_{1}=c_{2}=c_{4}=c_{5}=c_{6}=c_{8}=0, c_{3}=-\frac{2}{3^{\frac{2}{3}}}, c_{7}=\frac{2}{3^{\frac{2}{3}}}$, the parameterization in (3.23) reduces to $\Phi(u, v)=\left(u, v, u^{\frac{2}{3}}-v^{\frac{2}{3}}\right)$, which is a parameterization given in $(i)$ of theorem $A$ of [18]. Since, we are forced to exclude the ("may be") cases for $p \neq 0$ in (3.21), it is very difficult to get all the parameterizations given in theorem $A$ of [18] from (3.23). Yet nonetheless, the parameterization obtained in (3.23) is altogether a new parameterization of affine minimal translation surfaces.

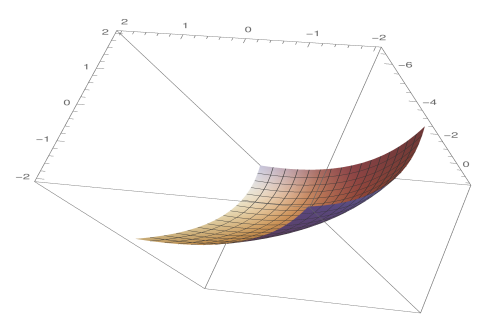

Figure 2. 3D plot of (3.23) for particular values of $c_{i} \in \mathbb{R}$.

\section{Translation surfaces satisfying $\Delta^{I} \Phi=\lambda_{i} \Phi$}

In this section, we classify translation surface in affine 3 -space satisfying the equation

$$
\Delta^{I} \Phi=\lambda_{i} \Phi_{i}
$$

where $\lambda_{i} \in \mathbb{R}, i=1,2,3$ and

$$
\Delta^{I} \Phi=\left(\Delta^{I} \Phi_{1}, \quad \Delta^{I} \Phi_{2}, \quad \Delta^{I} \Phi_{3}\right)
$$

where

$$
\Phi_{1}=u, \quad \Phi_{2}=v, \quad \Phi_{3}=\varphi(u)+\phi(v) .
$$

For the translation surface given by (3.1), the coefficients of the first affine fundamental form are given by (3.4). By a straightforward computation, the Laplacian operator $\Delta^{I}$ on $\mathbf{X}$ with the help of (4.2), (4.3), (3.4) and (2.10) turns out to be

$$
\Delta^{I} \Phi=\left(\frac{\varphi^{\prime \prime \prime}}{2 \varphi^{\prime \prime}}, \frac{\phi^{\prime \prime \prime}}{2 \phi^{\prime \prime}},-2+\frac{\varphi^{\prime} \varphi^{\prime \prime \prime}}{2 \varphi^{\prime \prime 2}}+\frac{\phi^{\prime} \phi^{\prime \prime \prime}}{2{\phi^{\prime \prime}}^{2}}\right) .
$$

Using (4.1) and (4.4), we have

$$
\begin{gathered}
\frac{\varphi^{\prime \prime \prime}}{2 \varphi^{\prime \prime^{2}}}=\lambda_{1} u, \\
\frac{\phi^{\prime \prime \prime}}{2 \phi^{\prime \prime^{2}}}=\lambda_{2} v, \\
-2+\frac{\varphi^{\prime} \varphi^{\prime \prime \prime}}{2 \varphi^{\prime \prime^{2}}}+\frac{\phi^{\prime} \phi^{\prime \prime \prime}}{2 \phi^{\prime \prime^{2}}}=\lambda_{3}(\varphi+\phi),
\end{gathered}
$$

where $\lambda_{1}, \lambda_{2}, \lambda_{3} \in \mathbb{R}$.

Definition 4.1. A surface in affine 3 -space is said to be $I$-harmonic if it satisfies the condition $\Delta^{I} \Phi=0$. 
We assume that $\mathbf{X}$ satisfies the condition $\Delta^{I} \Phi=0$. In this case from (4.5) and (4.6), we get

$$
\varphi^{\prime \prime \prime}=0, \quad \phi^{\prime \prime \prime}=0 .
$$

However, this is not solutions of (4.7), which implies that this case is not possible.

Theorem 4.2. Let $\boldsymbol{X}$ be a translation surface given by (3.1) in affine 3-space. There exists no translation surface satisfying the condition $\Delta^{I} \Phi=0$.

Combining (4.5), (4.6) and (4.7), we obtain

$$
-2+\varphi^{\prime} \lambda_{1} u-\lambda_{3} \varphi=p=\phi^{\prime} \lambda_{2} v+\lambda_{3} \phi,
$$

where $p \in \mathbb{R}$.

If $\lambda_{1} \neq 0, \lambda_{2}, \neq 0, \lambda_{3} \neq 0 \in \mathbb{R}$. By solving (4.9), we find

$$
\varphi(u)=c_{1} u^{\frac{\lambda_{3}}{\lambda_{1}}}-\frac{2+p}{\lambda_{3}}, \quad \phi(v)=c_{2} v^{\frac{\lambda_{3}}{\lambda_{2}}}+\frac{p}{\lambda_{3}},
$$

where $c_{1}, c_{2} \in \mathbb{R}$. Substituting (4.10) into (4.5) and (4.6), we can easily see that they do not satisfy these equations.

If $\lambda_{1} \neq 0, \lambda_{2}, \neq 0, \lambda_{3}=0 \in \mathbb{R}$, the equation (4.9) is reduced to

$$
-2+\varphi^{\prime} \lambda_{1} u=p=-\phi^{\prime} \lambda_{2} v .
$$

After solving (4.11), we get

$$
\varphi(u)=c_{1}+\frac{(2+p) \log u}{\lambda_{1}}, \quad \phi(v)=c_{2}-\frac{p \log v}{\lambda_{2}},
$$

where $c_{1}, c_{2} \in \mathbb{R}$. Substituting (4.12) into (4.5) and (4.6) yields $p=-1$. They satisfy these equations, thus the parametrization of $\mathbf{X}$ is given by

$$
\boldsymbol{\Phi}(u, v)=\left(u, v,\left(c_{1}+\frac{\log u}{\lambda_{1}}\right)+\left(c_{2}+\frac{\log v}{\lambda_{2}}\right)\right) .
$$

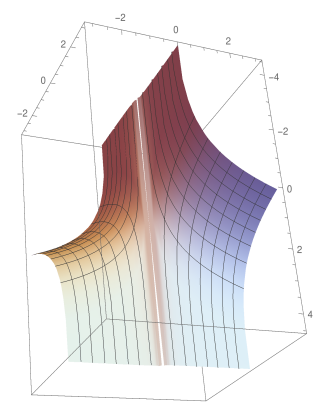

Figure 3. 3D plot of (4.13) for particular values of $c_{i}, \lambda_{i} \in \mathbb{R}$.

In the other cases with respect to constants $\lambda_{1}, \lambda_{2}$ and $\lambda_{3}$, the functions $\varphi(u)$ and $\phi(v)$ that are obtained from the solutions of the differential equation system (4.9), either contradicts the assumption of non-degeneracy or do not satisfy the equations (4.5) and (4.6). So, we have the following theorem:

Theorem 4.3. Let $\boldsymbol{X}$ be a non I-harmonic translation surface given by (3.1) in affine 3-space satisfying the condition $\Delta^{I} \Phi=\lambda_{i} \Phi_{i}, \lambda_{i} \in \mathbb{R}$, then it is an open part of the surface (4.13). 


\section{Translation surfaces satisfying $\Delta^{h} \Phi=\lambda_{i} \Phi$}

In this section, we classify the translation surfaces in affine 3 -space satisfying the equation

$$
\Delta^{h} \Phi=\lambda_{i} \Phi_{i}
$$

where $\lambda_{i} \in \mathbb{R}, i=1,2,3$ and

$$
\Delta^{h} \Phi=\left(\Delta^{h} \Phi_{1}, \quad \Delta^{h} \Phi_{2}, \quad \Delta^{h} \Phi_{3}\right) .
$$

For the affine translation surface given by (3.1), the coefficients of the Berwald-Blaschke metric of the translation surface or the second affine fundamental form are given by (3.5). By a straightforward computation, the Laplacian operator $\Delta^{h}$ on $\mathbf{X}$ with the help of (3.5), (4.3), (5.2) and (2.11) turns out to be

$$
\Delta^{h} \Phi=\left(\frac{\left(\varphi^{\prime \prime} \phi^{\prime \prime}\right)^{\frac{1}{4}} \varphi^{\prime \prime \prime}}{2 \varphi^{\prime \prime}}, \frac{\left(\varphi^{\prime \prime} \phi^{\prime \prime}\right)^{\frac{1}{4}} \phi^{\prime \prime \prime}}{2 \phi^{\prime \prime}}, \frac{\varphi^{\prime} \varphi^{\prime \prime \prime} \phi^{\prime{ }^{2}}+\varphi^{\prime \prime^{2}}\left(-4 \phi^{\prime{ }^{2}}+\phi^{\prime} \phi^{\prime \prime \prime}\right)}{2\left(\varphi^{\prime \prime} \phi^{\prime \prime}\right)^{\frac{7}{4}}}\right) .
$$

The equation (5.1) by means of (5.3) gives rise to the following system of ordinary differential equations:

$$
\begin{gathered}
\frac{\left(\varphi^{\prime \prime} \phi^{\prime \prime}\right)^{\frac{1}{4}} \varphi^{\prime \prime \prime}}{2 \varphi^{\prime{ }^{2}}}=\lambda_{1} u, \\
\frac{\left(\varphi^{\prime \prime} \phi^{\prime \prime}\right)^{\frac{1}{4}} \phi^{\prime \prime \prime}}{2{\phi^{\prime \prime 2}}^{2}}=\lambda_{2} v, \\
\frac{\varphi^{\prime} \varphi^{\prime \prime \prime} \phi^{\prime{ }^{2}}+\varphi^{\prime \prime 2}\left(-4{\phi^{\prime \prime}}^{2}+\phi^{\prime} \phi^{\prime \prime \prime}\right)}{2\left(\varphi^{\prime \prime} \phi^{\prime \prime}\right)^{\frac{7}{4}}}=\lambda_{3}(\varphi+\phi) .
\end{gathered}
$$

The equation (5.6) is reduced to

$$
-2\left(\varphi^{\prime \prime} \phi^{\prime \prime}\right)^{\frac{1}{4}}+\varphi^{\prime} \frac{\left(\varphi^{\prime \prime} \phi^{\prime \prime}\right)^{\frac{1}{4}} \varphi^{\prime \prime \prime}}{2 \varphi^{\prime \prime 2}}+\phi^{\prime} \frac{\left(\varphi^{\prime \prime} \phi^{\prime \prime}\right)^{\frac{1}{4}} \phi^{\prime \prime \prime}}{2 \phi^{\prime \prime}}=\lambda_{3}(\varphi+\phi) .
$$

First of all, we assume that $\mathbf{X}$ satisfies the condition $\Delta^{h} \Phi=0$. We call a surface satisfying this condition a $h$-harmonic surface. In this case from (5.3), we get

$$
\frac{\left(\varphi^{\prime \prime} \phi^{\prime \prime}\right)^{\frac{1}{4}} \varphi^{\prime \prime \prime}}{2 \varphi^{\prime \prime 2}}=0, \frac{\left(\varphi^{\prime \prime} \phi^{\prime \prime}\right)^{\frac{1}{4}} \phi^{\prime \prime \prime}}{2 \phi^{\prime \prime}}=0, \frac{\varphi^{\prime} \varphi^{\prime \prime \prime} \phi^{\prime \prime}+\varphi^{\prime \prime 2}\left(-4 \phi^{\prime \prime}{ }^{2}+\phi^{\prime} \phi^{\prime \prime \prime}\right)}{2\left(\varphi^{\prime \prime} \phi^{\prime \prime}\right)^{\frac{7}{4}}}=0 .
$$

The common solutions of the above three equations are $\varphi^{\prime \prime}=0$ and $\phi^{\prime \prime}=0$. But this is a contradiction with our assumption of the non-degenerate property of the surface $\mathbf{X}$. Thus, we can state:

Theorem 5.1. Let $\boldsymbol{X}$ be a translation surface given by (3.1) in affine 3-space, then there is no such translation surface satisfying the condition $\Delta^{h} \Phi=0$.

From (5.4) and (5.5), we see that

$$
\left(\varphi^{\prime \prime} \phi^{\prime \prime}\right)^{\frac{1}{4}}=\frac{2 \varphi^{\prime \prime^{2}} \lambda_{1} u}{\varphi^{\prime \prime \prime}}=\frac{2{\phi^{\prime \prime}}^{2} \lambda_{2} v}{\phi^{\prime \prime \prime}}
$$

Substituting (5.9) into (5.7), we have

$$
-4 \frac{\lambda_{1} u \varphi^{\prime \prime 2}}{\varphi^{\prime \prime \prime}}+\lambda_{1} u \varphi^{\prime}+\lambda_{3} \varphi=p=-\lambda_{2} v \phi^{\prime}+\lambda_{3} \phi
$$


or

$$
\lambda_{1} u \varphi^{\prime}+\lambda_{3} \varphi=p=-\lambda_{2} v \phi^{\prime}+\lambda_{3} \phi+4 \frac{\lambda_{2} v{\phi^{\prime \prime 2}}^{2}}{\phi^{\prime \prime \prime}},
$$

where $p \in \mathbb{R}$. When we try to solve the differential equation system (5.10) with respect to the constants $\lambda_{1}, \lambda_{2}, \lambda_{3}$, the following situations occur.

Case 1: If $\left\{\lambda_{1}=0, \lambda_{2}, \lambda_{3} \neq 0\right\},\left\{\lambda_{2}=0, \lambda_{1}, \lambda_{3} \neq 0\right\}$ and $\left\{\lambda_{1}=\lambda_{2}=0, \lambda_{3} \neq 0\right\}$, at least one of the functions $\varphi(u)$ or $\phi(v)$ is a constant. This is a contradiction to our assumption of the non-degenerate property of the surface $\mathbf{X}$.

Case 2: If $\left\{\lambda_{1}=0, \lambda_{2} \neq 0, \lambda_{3}=0\right\}$ and $\left\{\lambda_{1} \neq 0, \lambda_{2}=0, \lambda_{3}=0\right\}$, we again arrive at a contradiction.

Case 3: Let $\left\{\lambda_{1} \neq 0, \lambda_{2} \neq 0, \lambda_{3}=0\right\}$, from (5.10), we obtain

$$
-4 \frac{\lambda_{1} u \varphi^{\prime \prime 2}}{\varphi^{\prime \prime \prime}}+\lambda_{1} u \varphi^{\prime}=p=-\lambda_{2} v \phi^{\prime} .
$$

The general solution of $\phi(v)$ in (5.12) is given by

$$
\phi(v)=c_{1}+\frac{p \log u}{\lambda_{2}} .
$$

But the differential equation system (5.12) for the function $\varphi(u)$ cannot be solved analytically. In particular, if $p=0$, we have the function $\phi(v)$ is a constant and

$$
\varphi(u)=\frac{1}{2} c_{1}\left(3 u+c_{2}\right)^{\frac{3}{2}}+c_{3},
$$

where $c_{i} \in \mathbb{R}$. Again we get a contradiction.

Case 4: Let $\left\{\lambda_{1} \neq 0, \lambda_{2} \neq 0, \lambda_{3} \neq 0\right\}$, the differential equation system (5.10) for the function $\varphi(u)(p \neq 0, p=0)$ cannot be solved analytically.

Let's consider equations in (5.9) again, then we have

$$
\frac{2 \varphi^{\prime \prime} \lambda_{1} u}{\varphi^{\prime \prime \prime}}=p=\frac{2{\phi^{\prime \prime}}^{2} \lambda_{2} v}{\phi^{\prime \prime \prime}} .
$$

The general solution of (5.14) is given by

$$
\left\{\begin{array}{l}
\varphi(u)=c_{1}+c_{2} u-\frac{\sqrt{p}\left(u \arctan \left(\frac{u \sqrt{\lambda_{1}}}{\sqrt{p c_{3}}}\right)-\frac{\sqrt{p c_{3}} \log \left(\lambda_{1} u^{2}+p c_{3}\right)}{2 \sqrt{\lambda_{1}}}\right)}{\sqrt{c_{3} \lambda_{1}}}, \\
\phi(v)=c_{4}+c_{5} v-\frac{\sqrt{p}\left(v \arctan \left(\frac{v \sqrt{\lambda_{2}}}{\sqrt{p c_{6}}}\right)-\frac{\sqrt{p c_{6}} \log \left(\lambda_{2} v^{2}+p c_{6}\right)}{2 \sqrt{\lambda_{2}}}\right)}{\sqrt{c_{3} \lambda_{2}}}
\end{array}\right.
$$

for some constants $c_{i} \in \mathbb{R}$. Substituting (5.15) into (5.6), it does not satisfy. Thus, we have the following theorem:

Theorem 5.2. Let $\boldsymbol{X}$ be a non h-harmonic translation surface given by (3.1) in affine 3-space, then there is no such surface $\boldsymbol{X}$ satisfying the condition $\Delta^{h} \Phi=\lambda_{i} \Phi_{i}, \lambda_{i} \in \mathbb{R}$.

Note: It is to be noted here that the reader in this article have to encounter various times with a statementis not solvable analytically. By this, we mean that the differential equation is not solvable by any means and even sometimes if it may be, but the solution is complex. 


\section{Conclusion and future work}

Affine geometry is a very important topic in geometry, meanwhile we know that the study of "finite type of surfaces" is a very active area of research. There are lots of studies on "finite type" in various ambient spaces, in particular in Euclidean and Minkowskian spaces. As far as we know there is hardly any study discussing finite type of surfaces in affine spaces. So this paper serves as an initiative towards this direction. This study will help in more depth understanding the structural decomposition of position vector fields of surfaces in affine spaces. Finally, we would like to note some open problems, which can be potential problems for future research.

i A surface is called as a homothetical surface if it can be factored as as product of two independent smooth functions. The discussion of finite type of homothetical surfaces in affine spaces can be a problem of great interest.

ii There is a generalized class of surfaces known as revolution surfaces, one also can discuss these surfaces in this setting.

iii A linear Weingarten surface is a generalized class of constant mean and constant Gaussian curvature surfaces. So one can study those classes here also.

\section{References}

[1] M. Andrade, Calculus of affine structures and applications for isosurfaces (in portuguese), PhD dissertation, Rio de Janeiro, August, 2011.

[2] M. Andrade and T. Lewiner, Affine-invariant curvature estimators for implicit surfaces, Comput. Aided Geom. Des. 29, 162-173, 2012.

[3] K. Arslan, B. Bayram, B. Bulca and G. Ozturk, On translation surfaces in 4dimensional Euclidean space, Acta Comment. Univ. Tartu. Math. 20 (2), 123-133, 2016.

[4] M. Bekkar and B. Senoussi, Translation surfaces in the 3-dimensional space satisfying $\Delta^{I I I} r_{i}=\mu_{i} r_{i}$, J. Geom. 103 (3), 367-374, 2012.

[5] W. Blaschke, Vorlesungen über Differentialgeometrie II, Springer, Berlin, 1923.

[6] Ch. Baba-Hamed, M.Bekkar and H. Zoubir, Translation surfaces in the threedimensional Lorentz-Minkowski space satisfying $\Delta r_{i}=\lambda_{i} r_{i}$, Int. J. Math. Anal. 4 (17), 797-808, 2010.

[7] B.-Y. Chen, Total mean curvature and submanifolds of finite type, World Scientific, Singapore, 1984.

[8] F. Dillen, J. Pas and L. Vertraelen, On surfaces of finite type in Euclidean 3-space, Kodai Math. J. 13 (1), 10-21, 1990.

[9] O.J. Garay, An extension of Takahashis theorem, Geom. Dedicata, 34 (2), 105-112, 1990.

[10] W. Goemans, Surfaces in three-dimensional Euclidean and Minkowski space, in particular a study of Weingarten surfaces, PhD. Dissertation, September, 2010.

[11] E.F.C. Huamani, Affine Minimal surfaces with singularities, Masters dissertation, Rio de Janeiro, September, 2017.

[12] Y. Fu and Z.H. Hou, Affine translation surfaces with constant Gauss curvatures, Kyungpook Math. J. 50, 337-343, 2010.

[13] M.K. Karacan, D.W. Yoon and B. Bukcu, Translation surfaces in the three dimensional simply isotropic space $\mathbb{I}_{3}^{1}$, Int. J. Geom. Methods Mod. Phys. 13 (7), 1650088, 9 pp., 2016.

[14] A.M. Li, U. Simon and G. Zhao, Global affine differential geometry of hypersurfaces, De Gruyter Expositions Math., 328 pages, 1993.

[15] M. Magid and L.Vrancken, Affine translation surfaces, Results Math. 35, 134-144, 1999.

[16] F. Manhart, Die Affinminimalrückungsfl̈̈chen, Arch. Math. 44, 547-556, 1985. 
[17] U. Simon, A. Schwenk-Schellschmidt and H. Viesel, Introduction to the affine differential geometry of hypersurfaces. Lecture Notes, Science Univ. Tokyo Press, 1991.

[18] H. Sun, On affine translation surfaces of constant mean curvature, Kumamoto J. Math. 13, 49-57, 2000.

[19] Y. Yang, Y.H. Yu and H.L. Liu, Linear Weingarten centroaffine translation surfaces in $\mathbb{R}^{3}$, J. Math. Anal. Appl. 375, 458-466, 2011.

[20] D. Yanga and Y. Fu, On affine translation surfaces in affine space, J. Math. Anal. Appl. 440, 437-450, 2016.

[21] D.W. Yoon, Some Classification of translation surfaces in Galilean 3-space, Int. J. Math. Anal. 6 (28), 1355-1361, 2012. 